\title{
Chemical evolution models for local group dwarf spheroidal galaxies: the evolution of Fe-peak elements
}

\author{
Gustavo A. Lanfranchi ${ }^{1}$, Francesca Matteucci ${ }^{2}$ and Gabriele Cescutti ${ }^{2}$ \\ ${ }^{1}$ Núcleo de Astrofísica Teórica, Universidade Cruzeiro do Sul, \\ R. Galvão Bueno 868, Liberdade, 01506-000, São Paulo, SP, Brazil \\ email: gustavo.lanfranchi@cruzeirodosul.edu.br \\ ${ }^{2}$ Dipartimento di Astronomia-Universitá di Trieste, \\ Via G. B. Tiepolo 11, 34131 Trieste, Italy
}

\begin{abstract}
The evolution of Fe-peak elements of several Local Group Dwarf Spheroidal Galaxies are discussed based on the comparison between a chemical evolution model and obsevations. In our scenario, the evolution of these galaxies are mainly controlled by a low star formation efficiency coupled with very intense galactic winds. The low star formation rate gives rise to the observed low metallicities and to $[\mathrm{alpha} / \mathrm{Fe}]$ and $[\mathrm{s} / \mathrm{Fe}]$ ratios below solar, whereas the intense galactic winds are responsible for the sharp decrease observed in several abundance ratios. The shape of the stellar metallicity distributions are defined by both parameters and the observed data cannot be reproduced without evoking galactic winds. The same scenario applied to a standard model fits very well several Fe-peak elements, with different nucleosynthesis prescriptions for each set of elements.
\end{abstract}

Keywords. stars: abundances, galaxies: dwarf, Local Group

\section{The model}

We use the chemical evolution model for Dwarf Spheroidal (dSph) galaxies as described in Lanfranchi \& Matteucci (2004). Each galaxy model is specified by the prescriptions of the star formation (SF) and by the galactic wind (GW) efficiency chosen to reproduce the main features of these galaxies. The main characteristics of the models are the following: one zone with instantaneous and complete mixing of gas inside this zone; the stellar lifetimes are taken into account; the SFR is proportional to the gas mass and given by its efficiency $(\nu)$; the wind efficiency $\left(w_{i}\right)$ is proportional to the SFR. In the nucleosynthesis prescriptions we adopted the yields of van den Hoeck \& Groenewegen (1997) for low and intermediate mass stars, Nomoto et al. (1997) for type Ia supernovae and Woosley \& Weaver (1995) for massive stars.

\section{Results}

The abundance ratios of Fe-peak elements observed in dSph galaxies are compared to a standard model for these galaxies (Lanfranchi, Matteucci and Cescutti 2008). The main aim of such model is to draw a general scenario, without taking into account the particularities of each system, by putting all the data together in the comparison with the predictions of the model. A model capable of reproducing several other observational constraints, can also shed some light into the nucleosynthesis of Fe-peak elements. The SF proceeds in one long episode of activity ( $8 \mathrm{Gyr})$ with low efficiency $\left(\nu=0.3 \mathrm{Gyr}^{-1}\right)$. When the thermal energy of the gas equals or exceeds the binding energy of the galaxy, 


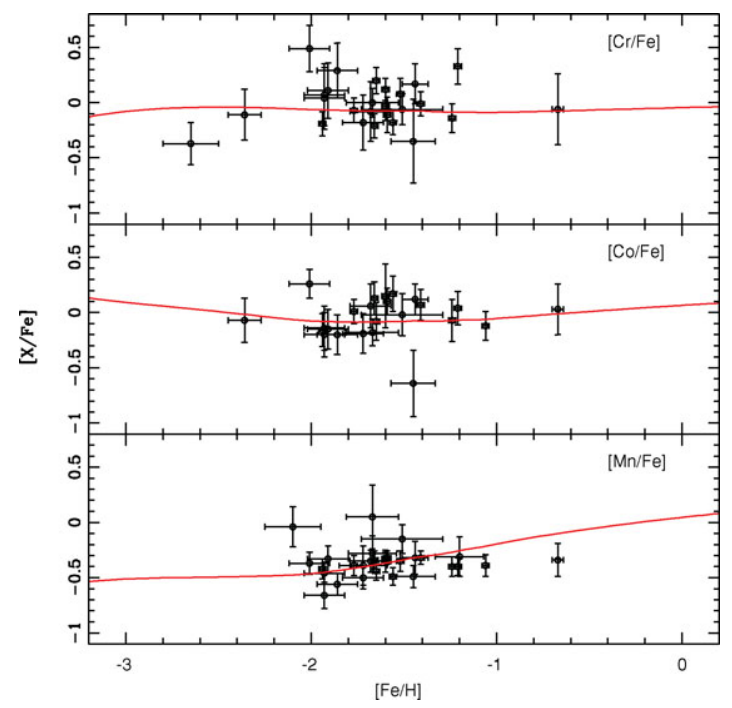

Figure 1. $[\mathrm{Cr}, \mathrm{Co}, \mathrm{Mn} / \mathrm{Fe}]$ vs. $[\mathrm{Fe} / \mathrm{H}]$ observed in dSphs compared to the predictions of the standard model.

an intense galactic wind $\left(\mathrm{w}_{i}=10\right)$ develops, removing a large fraction of the gas content of the galaxy. The SFR decreases then substantially, almost halting the formation of new alpha and r-process elements and of stars with metallicities higher than the one of the ISM when the wind began. This scenario allows the model to reproduce very well a few observational constraints: [alpha/Fe], [Fe-peak/Fe], and [s-r/Fe] ratios, the total mass, the gas mass and the stellar metallicity distributions. In Figure 1 the predictions of the standard model for $\mathrm{Cr}, \mathrm{Co}$, and $\mathrm{Mn}$ are compared to the observations (Shetrone et al. 2001, 2003, Sadakane et al. 2004, Geisler et al. 2005) with a very good agreement. The same model, which reproduces very well the evolution of alpha and neutron capture elements for the set of dSph as a whole (LM03, LMC08), fits the fe-peak elements, however with different yields for different elements. Whereas for $\mathrm{V}, \mathrm{Cr}$, and $\mathrm{Mn}$, we adopted the metallicity dependent yields of WW95, for Ti, Sc, Co, Zn, and Ni the WW95 yields with solar metallicity are used. The minor offsets in the plots are caused by the particularities in the SFHs and SFRs of each galaxy. Because of the differences in the evolution of each system, one should interprete these plots not as evolutionary diagrams: they only suggest a trend in the evolution of these elements instead of tracing the evolution.

\section{References}

Geisler D., Smith V. V., Wallerstein G., Gonzalez G., \& Charbonnel C. 2005, AJ, 129,1428

Lanfranchi, G. \& Matteucci, F. 2003, MNRAS, 345, 71

Lanfranchi, G. \& Matteucci, F. 2004, MNRAS, 351, 1338

Lanfranchi, G., Matteucci, F., \& Cescutti, 2008,A\&A, 481, 635

Nomoto, K., et al. 1997, Nucl. Phys. A, 616, 79

Sadakane, K., et al. 2004, PASJ, 56, 1041

Shetrone, M., Cote, P., \& Sargent, W. L. W. 2001, ApJ, 548,59

Shetrone, M., Venn, K. A., Tolstoy, E., \& Primas, F. 2003, AJ,125, 684

van den Hoeck, L. B. \& Groenwegen, M. A. T. 1997, A\&AS, 123, 305

Woosley, S. E. \& Weaver, T. A. 1995, ApJS, 101, 181 